\title{
Cancer-associated fibroblast and M2 macrophage markers together predict outcome in colorectal cancer patients
}

\author{
Mercedes Herrera, ${ }^{1,7}$ Alberto Herrera, ${ }^{1,7}$ Gemma Domínguez, ${ }^{1}$ Javier Silva, ${ }^{1}$ Vanesa García, ${ }^{1}$ José M. García, ${ }^{1}$ \\ Irene Gómez, ${ }^{1}$ Beatriz Soldevilla, ${ }^{1}$ Concepción Muñoz, ${ }^{2}$ Mariano Provencio, ${ }^{1}$ Yolanda Campos-Martin, ${ }^{3}$ \\ Antonio García de Herreros, ${ }^{4}$ Ignacio Casal, ${ }^{5}$ Félix Bonilla ${ }^{1,6}$ and Cristina Peña ${ }^{1,6}$ \\ ${ }^{1}$ Department of Medical Oncology, Puerta de Hierro Majadahonda University Hospital, Majadahonda, Madrid; Departments of ${ }^{2}$ Gastroenterology;

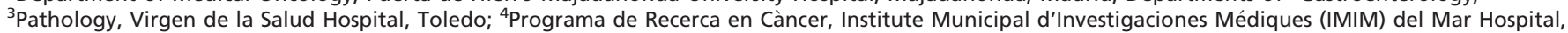 \\ Barcelona; ${ }^{5}$ Functional Proteomics Laboratory, Centro de Investigaciones Biológicas (CIB-CSIC), Madrid, Spain
}

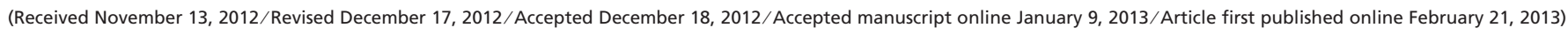

Tumor epithelial cells within a tumor coexist with a complex microenvironment in which a variety of interactions between its various components determine the behavior of the primary tumors. Cancer-associated fibroblasts (CAF) and M2 macrophages, characterized by high expression of different markers, including $\alpha$-SMA, FSP1 and FAP, or CD163 and DCSIGN, respectively, are involved in the malignancy of different tumors. In the present study, expression of the above markers in CAF and M2 macrophages was analyzed using RT-PCR and immunohistochemistry in the normal mucosa and tumor tissue from a cohort of 289 colorectal cancer patients. Expression of CAF and M2 markers is associated with the clinical outcome of colorectal cancer patients. Moreover, the combination of CAF and M2 markers identifies three groups of patients with clear differences in the progression of the disease. This combined variable could be a decisive factor in the survival of advanced-stage patients. Taken together, these analyses demonstrate the prognostic involvement of interrelationships between DCSIGN, CD163, $\alpha$-SMA, FSP1 and FAP markers in the survival of colon cancer patients. (Cancer Sci 2013; 104: 437-444)

olorectal cancer $(\mathrm{CC})$ is one of the most common diseases in Western populations. ${ }^{(1)}$ Currently, in clinical practice, although various biological and molecular markers have been suggested as useful tools for determining treatment and prognosis, the parameter most closely linked to the survival of patients is the stage of the disease.

Molecular characteristics relating to prognosis are usually linked to features of carcinoma cells. Thus, the level of carcinoembryonic antigen, which today is the most informative serum marker, depends on its expression in carcinoma cells. ${ }^{(2)}$

However, tumor epithelial cells within a tumor coexist with a complex microenvironment or stroma. ${ }^{(3)}$ In this complex scenario, a variety of interactions between its various components takes place. These might determine a series of events, such as tumor cell proliferation, metastatic potential and location of metastases. ${ }^{(4,5)}$

Fibroblasts are one of the most active cell types of the stroma. ${ }^{(6)}$ Fibroblasts are present in the stroma of normal tissues, where they perform tissue repair functions under certain physiological conditions, and in the stroma of tumors, in which they might represent the main component. ${ }^{(7)}$ They have been given various names: tumor-associated fibroblasts, carcinomaassociated fibroblasts (CAF) or myofibroblasts. ${ }^{(8)}$ In this state of permanent activation, fibroblasts can promote tumor growth and tumor progression, favoring a variety of tumor-specific mechanisms, ${ }^{(9)}$ which can influence the behavior of tumors and the prognosis of patients. ${ }^{(10)}$ These activated fibroblasts can be characterized molecularly by several markers that should be expressed by the fibroblasts in their activated state. Some of the most common CAF markers are $\alpha$-smooth muscle actin ( $\alpha$-SMA), fibroblast-specific protein 1 (FSP1 or S100A4) and fibroblast activation protein (FAP). ${ }^{(6,7)}$ It has been suggested that individually these markers could identify specific subpopulations of fibroblasts and that it would be more appropriate to use several markers to select the largest possible population of tumor fibroblasts. ${ }^{(11)}$

Inflammatory states can be associated with a risk for the development and progression of cancers. ${ }^{(12)}$ Tumor-associated macrophages can be found in the tumor in two phenotypically different populations: (i) M1 macrophages that will develop an antitumor response by activating the immune system and producing reactive oxygen species, nitric oxide and tumor necrosis factor; and (ii) M2 macrophages that will perform immunosuppressive functions and tumor promotion, ${ }^{(13)}$ as well as implementing the metastatic process by promoting angiogenesis and degradation of the extracellular matrix. ${ }^{(14)}$ This binary balance between M1/M2 cells has been verified using gene profiles, which support this phenotypic and functional change. ${ }^{(15)}$ The difference between M1 and M2 macrophages has been defined based on the cytokine production of IL-10 and IL-4/IL-13, respectively. ${ }^{(16-18}$ In addition, different markers are used to distinguish M1 and M2 macrophages. Thus, expression of CD163, or the most recently described, DCSIGN, are characteristics of M2 macrophages. ${ }^{16,17)}$ Despite the macrophage classification schemes, little is known about the complexity of the microenvironment populations of macrophages and their associations with clinical cancer progression.

Together with M2 macrophages, and as previously stated above, CAF are a large component of the stroma and generally tumor promoting. In this scenario, assessment of association between M2 plus CAF markers and survival of colon cancer patients is required. Results with prognostic implications, individually using some of the above markers, have been reported. ${ }^{(19-22)}$ The present study evaluates activation of fibroblasts and macrophages together using the analysis of CAF markers ( $\alpha$-SMA, FSP1 and FAP) and M2 macrophages (CD163 and DC-SIGN) to establish their relationship with clinical parameters and patient survival.

\footnotetext{
${ }^{6}$ To whom correspondence should be addressed.

E-mails: cpena@idiphim.org; felix.bonilla@salud.madrid.org

${ }^{7}$ These authors contributed equally to this work.
} 


\section{Materials and Methods}

Patients and samples. The present study is based on a consecutive series of 289 patients undergoing surgery for CC between January 2002 and December 2006. Informed written consent was obtained from all participants, as approved by the Research Ethics Board of Puerta de Hierro Majadahonda University Hospital. Tumor and normal colon mucosa (taken at least $3 \mathrm{~cm}$ from the outer tumor margin) were obtained immediately after surgery, snap-frozen in liquid nitrogen and stored at $-80^{\circ} \mathrm{C}$ until processing.

RNA extraction and real-time PCR. RNA extraction, target gene mRNA quantification and synthesis of cDNA were performed as previously described. ${ }^{(23)}$

An arbitrary value (0.01), corresponding to half the minimum value detected in the series, was assigned to the normal or tumor samples in which target gene expression was not detected, but expression of housekeeping was observed. Patients with normal or tumor samples without detection of housekeeping were excluded.

Immunohistochemistry analysis and antibodies. Anti-DCSIGN antibody [5D7] (Abcam, Cambridge, UK), anti-human CD163 (clone 10D6; Novocastra, Barcelona, Spain), anti-alpha smooth muscle actin antibody [1A4] (Abcam), anti-human S100A4 (DAKO, Glostrup, Denmark), peptide-affinity purified polyclonal antibody to fibroblast activation protein (FAP) alpha (Imgenex, San Diego, CA, USA) were used for cell immunostaining.

Procedures were performed as previously described. ${ }^{(24)}$

Immunohistochemistry data were evaluated as "low" or "high" expression, regarding the rate of positive cells for each sample and each marker. The analysis was evaluated by two independent pathologists.

Clinical-pathological characteristics, follow up and treatment of patients. The parameters obtained from the medical records of 289 patients were age, sex, tumor location, lymph node metastases, pathological stage (tumor-node-metastases classification), tumor histological grade, presence of polyposis in the surgical specimen and the presence of vascular invasion in tumors.

Colon cancer patients did not receive neo-adjuvant chemotherapy. Patients with rectal carcinoma who had received preoperative treatment with chemotherapy and radiotherapy or radiotherapy alone were excluded because of the difficulty of finding a suitable tumor for determining gene expression in these patients' surgical samples. Adjuvant treatment based on oxaliplatin (FOLFOX6, leucovorin $400 \mathrm{mg} / \mathrm{m}^{2}$ i.v. on day 1 as a 2 -h infusion, followed by 5 -fluorouracil bolus of $400 \mathrm{mg} / \mathrm{m}^{2}$ i.v. on day 1 , followed by $2400 \mathrm{mg} / \mathrm{m}^{2}$ i.v. 46-h infusion and oxaliplatin $100 \mathrm{mg} / \mathrm{m}^{2}$ i.v. as a 2 -h infusion on day 1) was administered to patients when clinically indicated and without medical contraindications who gave their written informed consent. ${ }^{(25)}$ Radiotherapy was also administered in rectal tumor cases.

Clinical follow up after surgery and diagnosis was based on periodic visits and clinical, biochemical and imaging techniques. Ultrasonic study was performed when liver function was impaired. Overall survival (OS) and disease-free survival (DFS) were defined as the period of time from diagnosis to death and the interval between diagnosis and first recurrence, respectively.

Statistical analysis. Spearman correlation was used to analyze correlation among different target genes. Protein and mRNA data association were contrasted using $t$-test following evaluation of equality of variance or not with Levene's test. Twotailed $P$-values $\leq 0.05$ were considered statistically different.

mRNA expression data of $\alpha$-SMA, FSP1, FAP, CD163 and DCSIGN were divided by tertiles $(33 \%$ and $66 \%)$ to get three groups of patients and develop the statistical analysis of
Table 1. Expression data of CAF and M2 macrophage markers studied in colorectal cancer patients

\begin{tabular}{lrrrrr}
\hline & DCSIGN & CD163 & \multicolumn{1}{c}{$\alpha$-SMA } & \multicolumn{1}{c}{ FSP1 } & \multicolumn{1}{c}{ FAP } \\
\hline$n$ & \multicolumn{1}{c}{239} & \multicolumn{1}{c}{235} & \multicolumn{1}{c}{270} & \multicolumn{1}{c}{264} & \multicolumn{1}{c}{208} \\
Median & 0.31 & 1.18 & 0.52 & 1.90 & 14.48 \\
Minimum & $<0.01$ & $<0.01$ & $<0.01$ & $<0.01$ & $<0.01$ \\
Maximum & 62.34 & 2227.07 & 522.47 & 642.67 & 299093.11 \\
33 percentile & 0.16 & 0.39 & 0.20 & 0.76 & 3.90 \\
66 percentile & 0.58 & 2.72 & 1.24 & 3.97 & 60.95 \\
\hline
\end{tabular}

CAF, cancer-associated fibroblast; FAP, fibroblast activation protein; FSP1, fibroblast-specific protein 1; SMA, smooth muscle actin.

Table 2. Correlation between the expression levels of M2 macrophage and CAF markers in colorectal cancer patients

\begin{tabular}{llllc}
\hline & CD163 & $\alpha$-SMA & FSP1 & FAP \\
\hline DCSIGN & $r=0.321 ;$ & $r=0.381 ;$ & $r=0.181 ;$ & $r=0.008 ;$ \\
& $P<0.001$ & $P<0.001$ & $P=0.007$ & $P=0.916$ \\
CD163 & & $r=0.460 ;$ & $r=0.257 ;$ & $r=0.419 ;$ \\
& & $P<0.001$ & $P<0.001$ & $P<0.001$ \\
$\alpha$-SMA & & $r=0.314 ;$ & $r=0.333 ;$ \\
& & $P<0.001$ & $P<0.001$ \\
FSP1 & & & $r=0.445 ;$ \\
& & & & $P<0.001$
\end{tabular}

CAF, cancer-associated fibroblast; FAP, fibroblast activation protein;

FSP1, fibroblast-specific protein 1; SMA, smooth muscle actin.

association with clinical-pathological parameters $\left(\chi^{2}\right.$ test) or OS and DFS (tests were carried out as previously described $\left.^{(23)}\right)$. In this way, patients between $0 \%$ and $33 \%$ fitted into the lower mRNA data expression of the target gene and were included in the "low expression group," patients between $33 \%$ and $66 \%$ were in the "medium expression group" and patients between $66 \%$ and $100 \%$ were in the "high expression group."

\section{Results}

Descriptive data of patient series and CAF and M2 macrophage markers. A large cohort of 289 tumor and normal counterpart tissue samples from 289 CC patients was studied. The patients included 179 males and 110 females. Eighteen cases were classified as pathological stage I, 157 were stage II, 100 were stage III and 14 stage IV. The series was followed for a mean of 5 years (range of patient follow ups: 1-187 months). Of 275 patients with known data, 86 recurrences were recorded during this period. The number of exitus observed during the period was 88 . The number of patients in which detection of the target gene was possible and generic data of each gene mRNA quantification is detailed in Table 1.

Spearman's test revealed a direct correlation between macrophage and CAF markers (Table 2). In parallel, correlation among CAF and M2 macrophage markers was observed, excluding FAP and DCSING (Table 2).

Sixty tumor samples and the normal counterpart were analyzed for DCSIGN, CD163, $\alpha$-SMA, FSP1 and FAP stromal protein expression. Stromal target protein expression was evaluated as low or high levels and its association with mRNA expression levels was analyzed (Fig. 1). Statistical association was observed between mRNA expression levels and protein levels of FSP1 and FAP. In addition, a clear trend toward statistical association was found between protein and mRNA expression levels of DCSIGN, CD163 and $\alpha$-SMA (Fig. 1B).

CAF and M2 markers are associated with patients' clinical characteristics. Statistical association between high mRNA levels 
(A)
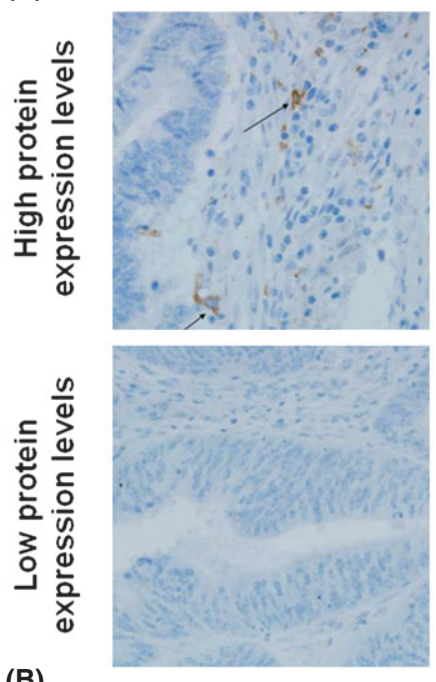

(B)

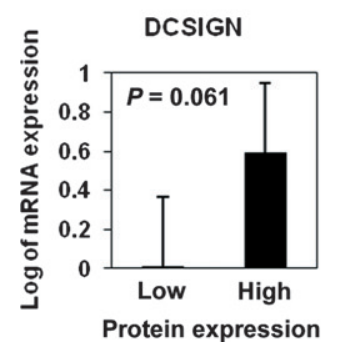

CD163
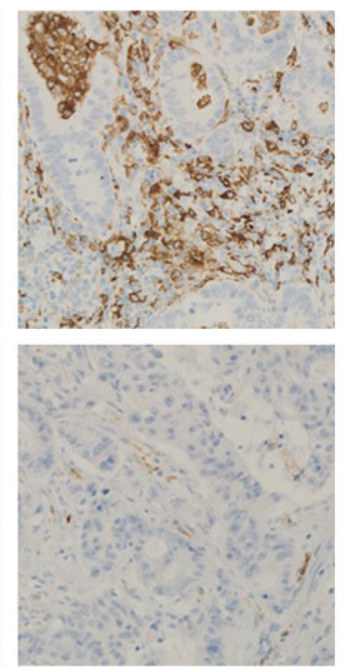

ASMA
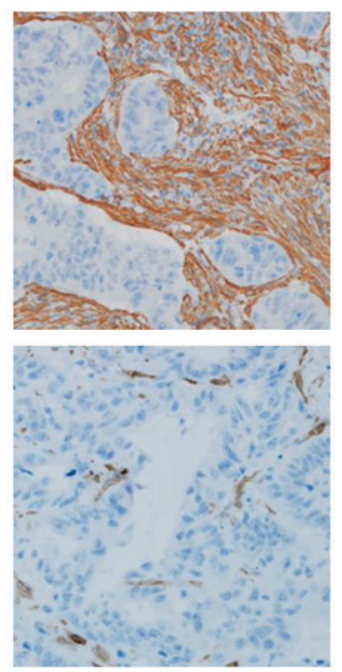

FSP1
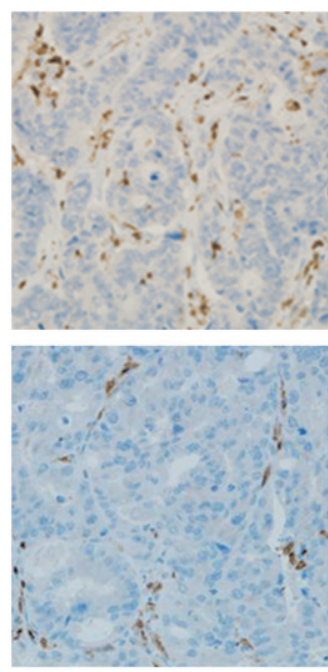

FAP
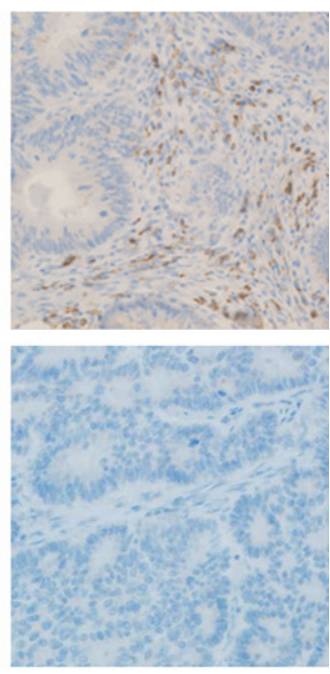
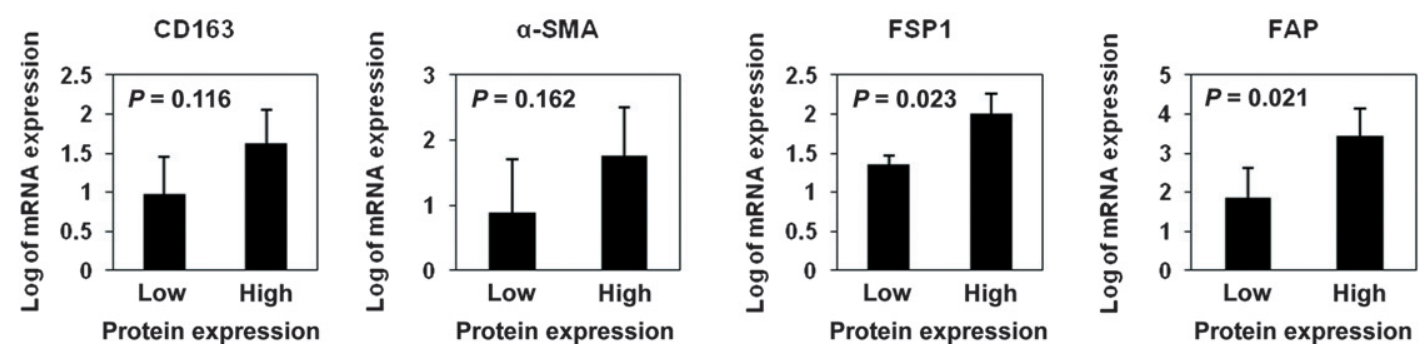

Fig. 1. mRNA quantitative expression versus immunohistochemistry. (A) DCSIGN, CD163 M2, $\alpha$-smooth muscle actin ( $\alpha$-SMA), fibroblast-specific protein 1 (FSP1) and fibroblast activation protein (FAP) immunohistochemistry. Representative pictures of high and low protein expression levels of DCSIGN, CD163 M2 macrophage and $\alpha$-SMA, FSP1 and FAP cancer-associated fibroblast (CAF) markers in human tumor samples. Arrows indicate positive cells. Original magnification, $\times 20$. (B) Association between protein and mRNA expression levels. Statistical association between protein and mRNA expression levels of FSP1 and FAP, CAF markers. In addition, a clear trend toward statistical association is observed between protein and mRNA expression levels of DCSIGN and CD163, M2 macrophage markers and the $\alpha$-SMA, CAF marker. Bar charts show the average ( \pm confidence interval) of mRNA expression levels of each gene in the low and high protein expression groups.

of CD163, a-SMA and FSP-1 and recurrence was observed $(P=0.023, P=0.028$ and $P=0.012$, respectively), while only a trend toward statistical association was found for FAP $(P=0.083)$. In addition, CD163 was also statistically associated with exitus $(P=0.029)$ and a trend linking $\alpha$-SMA, FSP1 and FAP with exitus was found $(P=0.154, P=0.067$ and $P=0.143$, respectively).

No relationship was found between the stromal markers analyzed and other clinical-pathological variables, including the sex of patients, tumor location, histological degree, vascular invasion, polyposis, lymph node metastases and pathological stage (data not shown).

CAF and M2 macrophage markers are associated with DFS and OS. Statistical association between DFS and the mRNA expression levels of $\alpha$-SMA and FSP-1, CAF markers and CD163, and the M2 macrophage marker was observed (Fig. $\mathrm{S} 1 \mathrm{~A}, \mathrm{~B})$. There was also a trend between FAP expression levels and DFS (Fig. S1A). Kaplan-Meier analysis revealed similar behavior of different gene expression data tertiles of each gene. Therefore, to simplify the analysis, those tertiles were grouped according to mathematical behavior as "high" and "low" expression of the target gene (Fig. S1). The new analysis with these two categories improves the prognostic value for CD163, a-SMA, FSP-1 and FAP (Fig. 2).

CD163 mRNA expression levels were statistically associated with OS in CC patients (Fig. 3A). There was a statistical association between the expression levels of FSP-1 and OS (Fig. 3C). Patients whose tumors revealed high expression levels of $\alpha$-SMA or FAP showed a trend towards shorter OS (Fig. 3B,D).

Together, these results indicate the clinical meaning of a-SMA, FSP1 and FAP, CAF markers and CD163, a M2 macrophage marker, as all of them showed a clear association with cancer patients' survival.

No association between DCSIGN, and DFS or OS was found. The dichotomization of the variable, based on mathematical behavior, did not show any association either (Fig. $\mathrm{S} 1 \mathrm{~B}, \mathrm{C})$. However, this variable was included in further analysis as Cox analysis demonstrated its usefulness, also indicating its involvement in clinical cancer prognosis.

Combination of CAF and M2 macrophage markers predicts DFS and OS. To study the possible additive effect of different markers expression, the patients were classified in different groups according to the number of CAF and M2 markers highly expressed. Thus, patients were first classified based on the expression of zero, one, two or three CAF markers and expression of zero, one or two M2 markers. Then, association with DFS was established $(P=0.009$ and $P=0.030$, respectively).

Next, to dichotomize the analysis, and based on similar mathematical behavior, patients with zero, one or two CAF markers were grouped as "low-rate CAF" versus those with three markers of expression, "high-rate CAF." Likewise, the "low-rate M2" group included no expression of any marker and the "high-rate M2" group had expression of one or two M2 macrophage markers. Previously observed correlation between DFS or OS was confirmed (CAF markers: $P=0.004$ 

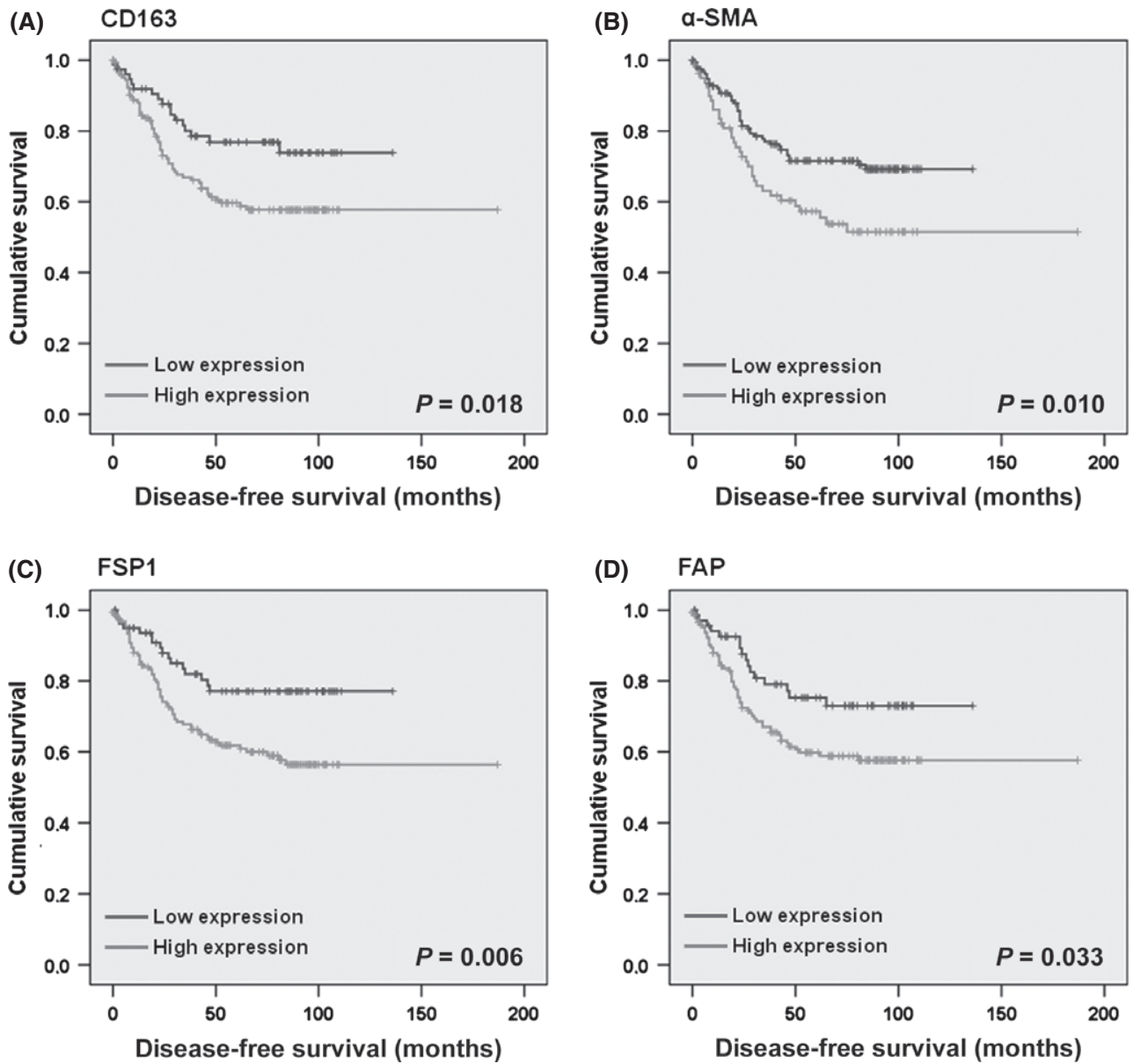

Fig. 2. Kaplan-Meier curves between expression levels of cancer-associated fibroblast (CAF) and M2 macrophage markers (individually) and disease-free survival (DFS) in colorectal cancer patients. Association between CD163, a M2 macrophage marker (A), and $\alpha$-smooth muscle actin $(\alpha-S M A)(B)$, fibroblast-specific protein 1 (FSP1) (C) and fibroblast activation protein (FAP) (D) CAF markers with DFS. "Low expression" refers to a low-expression tertile for $\alpha$-SMA and low- and medium-expression tertiles for CD163, FSP1 and FAP.

and $P=0.038 ; \mathrm{M} 2$ markers: $P=0.009$ and $P=0.015$, for DFS and OS, respectively).

Finally, expression of CAF and M2 markers were combined and patients classified as "basal" (low-rate CAF and M2 markers), "middle" (low-rate CAF markers and high-rate M2 markers or vice versa) or "solid stromal expression" (high-rate CAF and M2 markers). This classification was then used for further studies. Statistical analysis showed that the rate of CAF-M2 markers also correlated with DFS and OS (Fig. 4A,B, respectively).

In parallel, CAF-M2 markers, together with vascular invasion, histological degree and pathological stage were determined as independent prognostic variables for DFS (Table 3) and $\mathrm{CAF}-\mathrm{M} 2$ markers, vascular invasion and pathological stage were for OS (Table 3).

CAF-M2 markers' association with DFS and OS according to the pathological stage localization of the tumors. The prognostic value of CAF-M2 markers in different tumor stages was also studied. The analysis showed that the expression levels of our CAF-M2 markers correlated with DFS in early $(P=0.052)$ and advanced $(P=0.050)$ stages (Fig. $4 \mathrm{C})$. Nevertheless, OS analysis showed that the studied markers tended to show a prognosis value in stages III and IV $(P=0.069)$ (Fig. 4D), but not in earlier stages $(P=0.596)$.

Because the results suggested that the studied markers behave as a better predictive variable in the advanced stage for
OS, the multivariate models were rechecked stratifying the series for stage status. This analysis confirmed that the studied variable CAF-M2 markers interacts with the pathological stage, giving a prognostic value for OS in patients in stage III or IV (Table 4).

Finally, we were interested in determining whether the CAF-M2 markers variable behaved differently regarding anatomical tumor area. Survival analysis revealed clear differences in DFS and OS for patients with colon tumors only (Fig. 4E,F, respectively). In contrast, involvement of the CAF-M2 marker variable in the survival of patients with rectal carcinomas was not observed.

\section{Discussion}

Cancer-associated fibroblasts contribute to a tumor-permissive neighborhood and M2 macrophages display immunosuppressive and tumor-promoting functions, including prometastatic function by production of angiogenic and extracellular matrix breakdown factors. ${ }^{(23)}$ Although some steps involved in the cross-talk between these cells are known, there is still a lot that is not clear, but if clarified could lay the foundations for the development of new treatment strategies that could be used to control the disease.

Although some evidence individually supports our findings on $\mathrm{CAF}$ markers in the prognosis of $\mathrm{CC},{ }^{(20-22)}$ very little is 

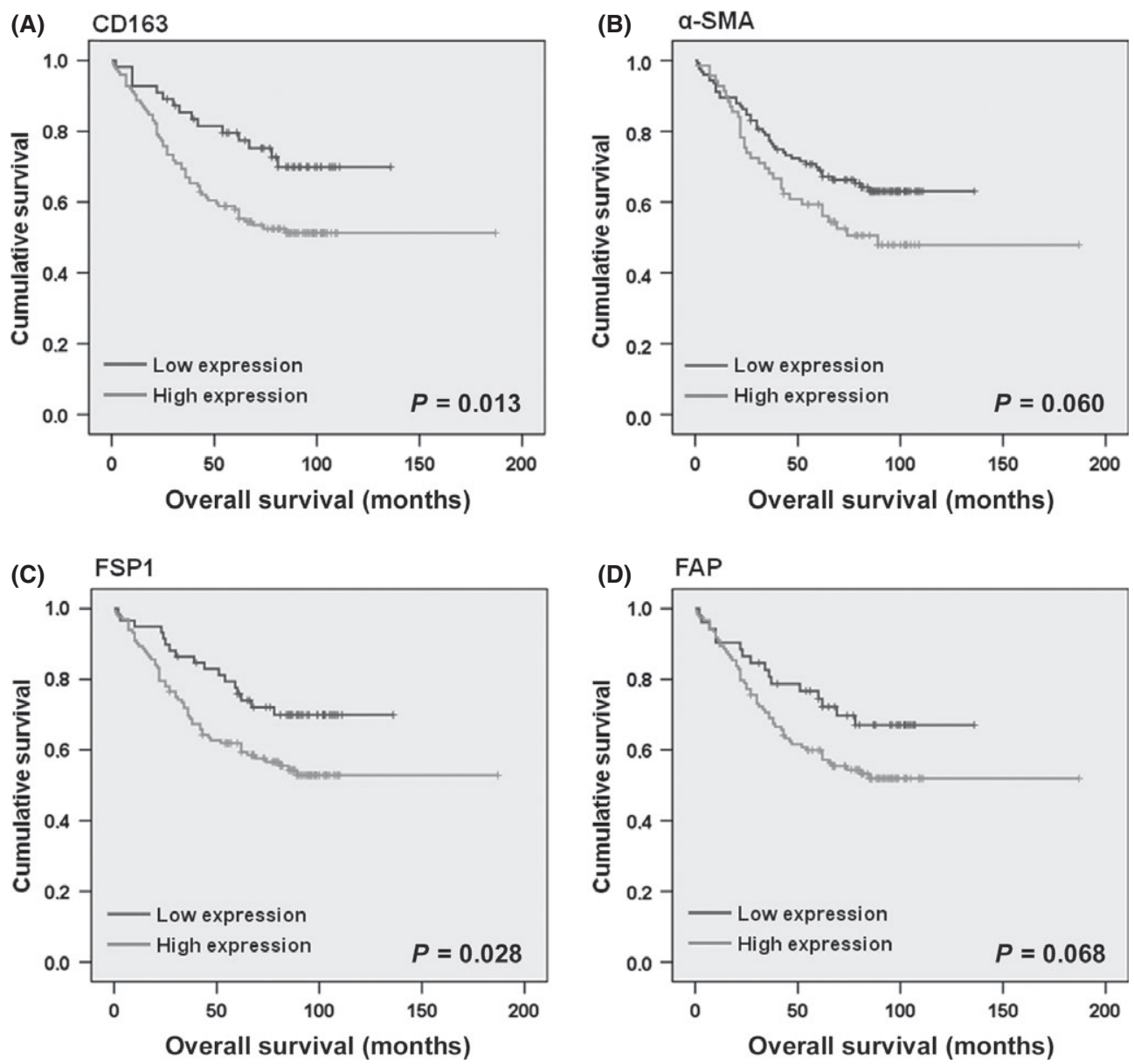

Fig. 3. Kaplan-Meier curves between expression levels of cancer-associated fibroblast (CAF) and M2 macrophage markers (individually) and overall survival in colorectal cancer patients. Association of CD163, a M2 macrophage marker (A), and fibroblast-specific protein 1 (FSP1) (C), a CAF marker, with overall survival. $\alpha$-Smooth muscle actin ( $\alpha$-SMA) (B) and fibroblast activation protein (FAP) (D) expression levels, CAF markers, showed a trend towards an association with overall survival.

known about M2 markers. In fact, infiltration of tumor-associated macrophages correlates in many cancers with a bad prognosis. However, it has also been reported that a greater abundance of macrophages in $\mathrm{CC}$ correlates with a more favorable prognosis. ${ }^{(26)}$ These contradictory data could be clarified with specific M1 and M2 markers. Thus, we observed that mRNA expression levels of CAF expression markers are related to poor DFS and OS, but that when the addition of M2 markers to survival analysis is taken into consideration, this relationship is more robust. Moreover, multivariate analysis clearly demonstrates that this variable, with both types of markers, is an individual prognostic marker that is statistically stronger than CAF markers or M2 markers individually.

Stromal gene expression profile assesses the difference between pathological stages in gastrointestinal cancers or between normal and tumor fibroblasts in colon cancer. However, these studies did not demonstrate prognostic involvement of the stromal gene expression profile. ${ }^{(27,28)}$ We think that our results can be more easily translated into clinical practice, therefore helping to identify patients with a worse prognosis. Even more interesting is the interaction between the expression of our five selected CAF and M2 markers and the pathological stage. As multivariate analysis shows, the effect of our studied markers is stronger in advanced stages for OS. Today, the available therapies based on chemotherapy and biological targeting strategies, administered as adjuvant treatment in stage
III or when the disease is metastatic, have increased both DFS and OS. Thus, these longer survivals are a favorable event that will permit us to see the positive relationship of our stromal markers and patient survival in advanced stages. In this context, it is possible that new therapies targeting microenvironment components could help improve the medical treatment of $\mathrm{CC}$, especially those with a worse outcome.

The lack of association between our five studied CAF and M2 markers and the prognosis in rectal cancer patients is also interesting. First, these patients were the smallest group in our cohort (16\% of the total series) and a random effect is possible. However, we could argue that rectal tumors behave differently from colon tumors. The treatment for rectal cancer is a modification of traditional colon cancer treatment, mainly because of the addition of radiotherapy combined with chemotherapy, administered preoperatively. However, the rectal carcinomas in our series received the treatment postoperatively, allowing us to obtain representative tumor samples for a correct molecular study. This could indicate that radiotherapy in rectal carcinomas modifies the behavior of the disease and, thus, the informative value of the molecular markers studied. However, it is also possible that in rectal carcinoma the stromal component has a different biological significance, whereby fibroblasts and M2 macrophages might play roles that are different from those observed in colon carcinomas. 
(A)

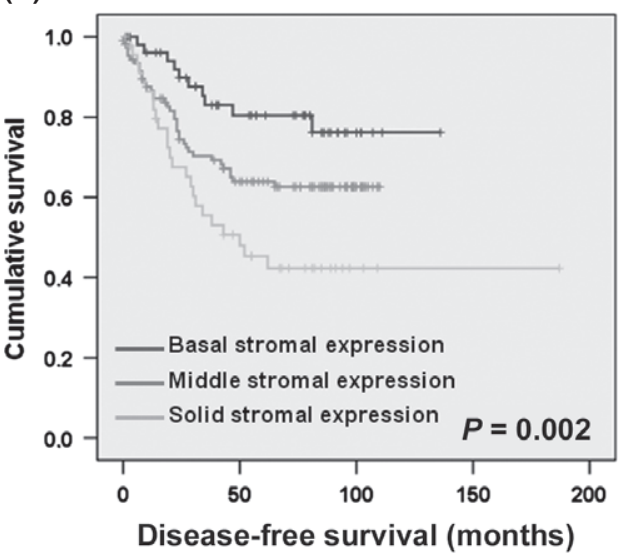

(C)

CAF-M2 markers

\section{Stages III and IV}

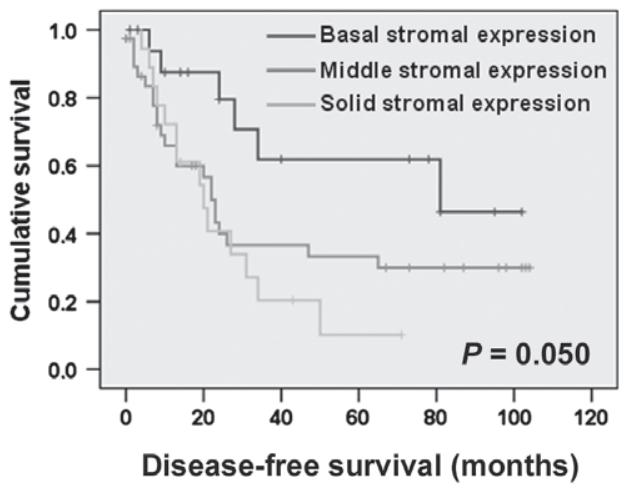

CAF-M2 markers

(E)

Colon location

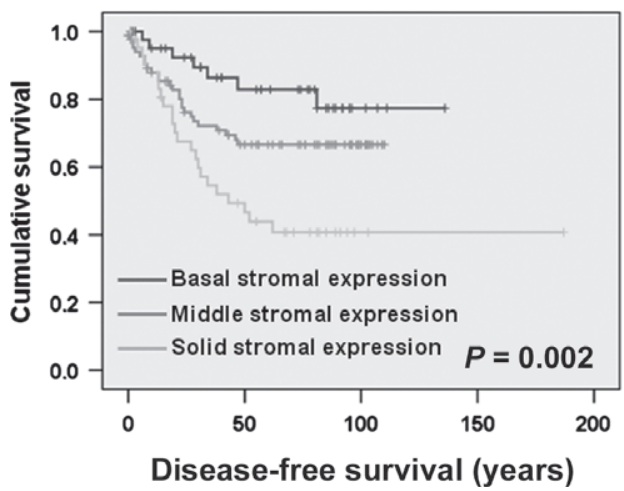

(B) CAF-M2 markers

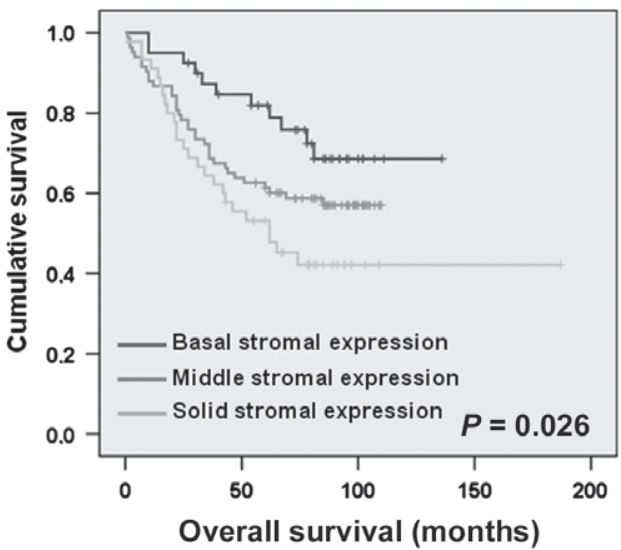

(D)

\section{CAF-M2 markers}

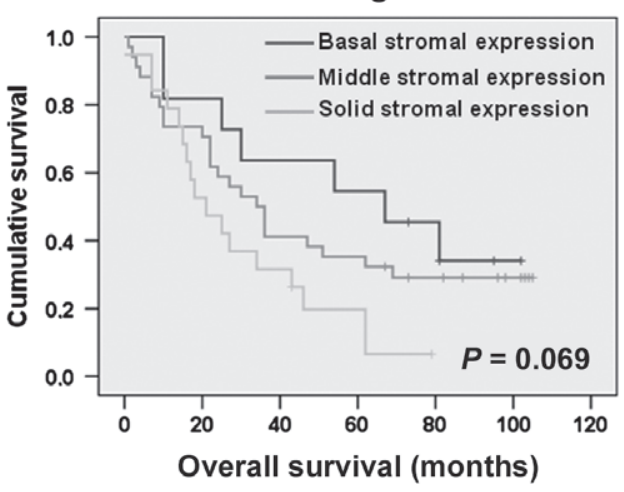

CAF-M2 markers

(F)

\section{Colon location}

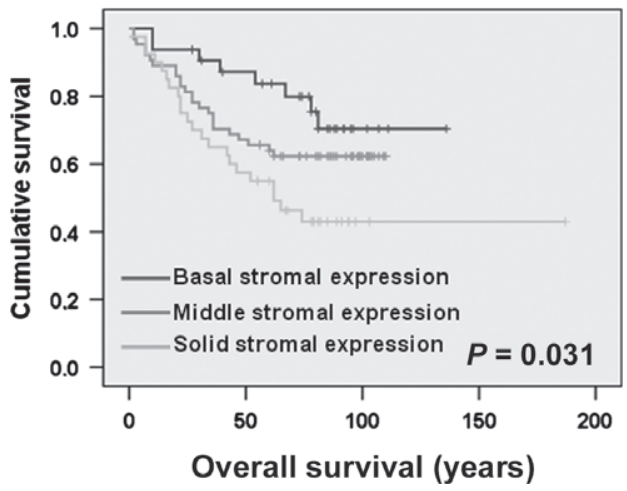

Fig. 4. (A,B) Kaplan-Meier curves between the expression levels of cancer-associated fibroblast (CAF) plus M2 macrophage markers and colorectal cancer (CC) patient survival. Association between the variable CAF plus M2 macrophage markers taken together and disease-free survival (DFS) (A) or overall survival (OS) (B). "Basal stromal expression" includes the CAF and M2 low-rate markers variable. "Middle stromal expression" comprises CAF high-rate markers and M2 low-rate markers or vice versa. "Solid stromal expression" contains CAF and M2 high-rate markers. $(C, D)$ Kaplan-Meier curves between the expression levels of CAF plus M2 macrophage markers and survival for the pathological stage in colorectal cancer (CC) patients. Association between the variable of CAF plus M2 macrophage markers taken together and DFS in patients with advanced pathological stages (C). A clear trend was observed in advanced pathological stages between CAF plus M2 markers and OS (D). (E, F) Kaplan-Meier curves between the expression levels of CAF plus M2 macrophage markers and survival in relation to tumor location in CC patients. A clear association was observed between CAF plus M2 macrophage markers and DFS in colon cancer patients (E). Similar results were found for OS; the association was observed in colon cancer patients (F).

The clinical meaning of the present study is indicated by the observation of the prognostic involvement of DCSIGN and CD163, M2 macrophage markers, a-SMA, FSP1 and FAP CAF markers, as well as their interrelationship in colon cancer patients' survival, particularly in those at advanced stages. Knowledge of the real involvement of these factors in the development of the disease encourages the search for new therapies that would target the stromal component of colorectal 
Table 3. Multivariate Cox analysis of the association between CAF-M2 markers and disease-free survival and overall survival of colorectal cancer patients

\begin{tabular}{|c|c|c|c|c|c|}
\hline & Variable & Category & $\mathrm{HR}$ & $95 \% \mathrm{Cl}$ & $P$-value \\
\hline \multirow[t]{8}{*}{$\begin{array}{l}\text { Disease-free } \\
\text { survival }\end{array}$} & $\begin{array}{l}\text { Vascular } \\
\text { invasion }\end{array}$ & $\begin{array}{l}\text { Yes vs } \\
\text { No }\end{array}$ & 1.775 & $1.045-3.015$ & 0.034 \\
\hline & Histological & & & & 0.082 \\
\hline & degree & II vs I & 1.381 & $0.781-2.443$ & 0.267 \\
\hline & & III vs I & 2.511 & $1.121-5.625$ & 0.025 \\
\hline & $\begin{array}{l}\text { Pathological } \\
\text { stage }\end{array}$ & $\begin{array}{l}\mathrm{III}+\mathrm{IV} v s \\
\mathrm{I}+\mathrm{II}\end{array}$ & 3.857 & $2.245-6.627$ & $<0.001$ \\
\hline & CAF-M2 & & & & 0.008 \\
\hline & & $\begin{array}{l}\text { Medium } \\
\text { rate } \\
\text { vs Low } \\
\text { rate }\end{array}$ & 2.219 & $1.093-4.503$ & 0.027 \\
\hline & & $\begin{array}{l}\text { High rate } \\
\text { vs } \\
\text { Low rate }\end{array}$ & 3.319 & $1.555-7.085$ & 0.002 \\
\hline \multirow[t]{5}{*}{$\begin{array}{l}\text { Overall } \\
\text { survival }\end{array}$} & $\begin{array}{l}\text { Vascular } \\
\text { invasion }\end{array}$ & Yes vs No & 1.649 & $0.979-2.778$ & 0.060 \\
\hline & $\begin{array}{l}\text { Pathological } \\
\text { stage }\end{array}$ & $\begin{array}{l}\text { III + IV vs } \\
I+I I\end{array}$ & 5.001 & $2.824-8.856$ & $<0.001$ \\
\hline & CAF-M2 & & & & 0.044 \\
\hline & & $\begin{array}{l}\text { Middle vs } \\
\text { Basal } \\
\text { stromal } \\
\text { expression }\end{array}$ & 1.564 & $0.792-3.086$ & 0.19 \\
\hline & & $\begin{array}{l}\text { Solid vs } \\
\text { stromal } \\
\text { expression }\end{array}$ & 2.445 & $1.185-5.045$ & 0.01 \\
\hline
\end{tabular}

$\mathrm{Cl}$, confidence interval; $\mathrm{HR}$, hazard ratio.

and other cancers and offer a synergistic effect with the current systemic therapies.

\section{References}

1 Jemal A, Siegel R, Ward E et al. Cancer statistics. CA Cancer J Clin 2006; 5: $106-30$.

2 Libutti SK, Saltz LB, Tepper JE. Colon Cancer. In: Libutti SK, Saltz LB, Tepper JE, eds. Principles \& Practice of Oncology, 8th edn. Philadelphia, PA: Lippincott Williams \& Wilkins, 2008; 1232-85.

3 Tlsty TD, Hein PW. Know thy neighbor: stromal cells can contribute oncogenic signals. Curr Opin Genet Dev 2001; 11: 54-9.

4 Bissell MJ, Radisky D. Putting tumours in context. Nat Rev Cancer 2001; 1 : 46-54.

5 Li H, Fan X, Houghton J. Tumor microenviroment: the role of the tumor stroma in cancer. J Cell Biochem 2007; 101: 805-15.

6 Xouri G, Christian S. Origin and function of tumor stroma fibroblasts. Semin Cell Dev Biol 2010; 21: 40-6.

7 Räsänen K, Vaheri A. Activation of fibroblasts in cancer stroma. Exp Cell Res 2010; 316: 2713-22.

8 Beacham DA, Cukierman E. Stromagenesis: the changing face of fibroblastic microenvironments during tumor progression. Semin Cancer Biol 2005; 15 329-41.

9 Orimo A, Weinberg RA. Stromal fibroblasts in cancer: a novel tumor-promoting cell type. Cell Cycle 2006; 5: 1597-601.

10 Gout S, Huot J. Role of cancer microenvironment in metastasis: focus on colon cancer. Cancer Microenviron 2008; 1: 69-83.

11 Sugimoto H, Mundel TM, Kieran MW, Kalluri R. Identification of fibroblast heterogeneity in the tumor microenvironment. Cancer Biol Ther 2006; 5 : 1640-6.

12 Qian BZ, Pollard JW. Macrophage diversity enhances tumor progression and metastasis. Cell 2010; 141: 39-51.

13 Allavena P, Sica A, Garlanda C, Mantovani A. The Yin-Yang of tumor-associated macrophages in neoplastic progression and immune surveillance. Immunol Rev 2008; 222: 155-61.
Table 4. Statistical interaction between CAF-M2 variable and tumor pathological stage regarding overall survival predictive outcome value in colorectal cancer patients

\begin{tabular}{|c|c|c|c|c|c|}
\hline & Variable & Category & HR & $95 \% \mathrm{Cl}$ & $P$-value \\
\hline \multirow[t]{5}{*}{$\begin{array}{l}\text { Overall } \\
\text { survival }\end{array}$} & $\begin{array}{l}\text { Vascular } \\
\text { invasion }\end{array}$ & Yes vs No & 1.743 & $1.024-2.966$ & 0.041 \\
\hline & $\begin{array}{l}\text { Pathological } \\
\text { stage }\end{array}$ & $\begin{array}{l}\text { III + IV vs } \\
I+I I\end{array}$ & 2.818 & $1.128-7.040$ & 0.027 \\
\hline & CAF-M2 * & & & & 0.035 \\
\hline & $\begin{array}{l}\text { Pathological } \\
\text { stage }\end{array}$ & $\begin{array}{l}\text { Stage * } \\
\text { Middle vs } \\
\text { Basal } \\
\text { stromal } \\
\text { expression }\end{array}$ & 1.574 & $0.675-3.669$ & 0.294 \\
\hline & & $\begin{array}{l}\text { Stage * Solid } \\
\text { vs Basal } \\
\text { stromal } \\
\text { expression }\end{array}$ & 2.951 & $1.204-7.232$ & 0.018 \\
\hline
\end{tabular}

$\mathrm{Cl}$, confidence interval; HR, hazard ratio.

\section{Acknowledgments}

M. Eaude is acknowledged for help with the English text. Members of the F. B. laboratory contributed with constructive criticism throughout this project. This work has been supported by Fundación Científica AECC, SAF2010-20750, S2010/BMD-2344, RTICC-RD06/0020/0020, PI12/02037 and Fundación Banco Santander. A.G.H. laboratory was supported by RD06/0020/0040. C.P. is the recipient of a Miguel Servet Contract from the Instituto de Salud Carlos III (CP09/00294) and V.G. is the recipient of a Fundación Científica AECC fellowship.

\section{Disclosure Statement}

The authors have no conflict of interest.

14 Mantovani A, Allavena P, Sica A, Balkwill F. Cancer-related inflammation. Nature 2008; 454: 436-44.

15 Ojalvo LS, King W, Cox D, Pollard JW. High-density gene expression analysis of tumor-associated macrophages from mouse mammary tumors. Am J Pathol 2009; 174: 1048-64.

16 Joyce JA, Pollard JW. Microenvironmental regulation of metastasis. Nat Rev Cancer 2009; 9: 239-52.

17 Domínguez-Soto A, Sierra-Filardi E, Puig-Kröger A et al. Dendritic cellspecific ICAM-3-grabbing nonintegrin expression on M2-polarized and tumor-associated macrophages is macrophage-CSF dependent and enhanced by tumor-derived IL-6 and IL-10. J Immunol 2011; 186: 2192-200.

18 Mosser DM, Edwards JP. Exploring the full spectrum of macrophage activation. Nat Rev Immunol 2008; 8: 958-69.

19 Nakayama Y, Nagashima N, Minagawa N et al. Relationships between tumor-associated macrophages and clinicopathological factors in patients with colorectal cancer. Anticancer Res 2002; 22: 4291-6.

20 Kwak JM, Lee HJ, Kim SH et al. Expression of protein S100A4 is a predictor of recurrence in colorectal cancer. World J Gastroenterol 2010; 16: 3897-904.

21 Tsujino T, Seshimo I, Yamamoto $\mathrm{H}$ et al. Stromal myofibroblasts predict disease recurrence for colorectal cancer. Clin Cancer Res 2007; 13: 2082 90.

22 Henry LR, Lee HO, Lee JS et al. Clinical implications of fibroblast activation protein in patients with colon cancer. Clin Cancer Res 2007; 13: 173641.

23 Gomez I, Peña C, Herrera M et al. TWIST1 is expressed in colorectal carcinomas and predicts patient survival. PLOS ONE 2011; 6: e18023.

24 Peña C, García JM, Larriba MJ et al. SNAI1 expression in colon cancer related with $\mathrm{CDH} 1$ and VDR downregulation in normal adjacent tissue. Oncogene 2009; 28: 4375-85.

25 Tournigand C, André T, Achille E et al. FOLFIRI followed by FOLFOX6 or the reverse sequence in advanced colorectal cancer: a randomized GERCOR study. J Clin Oncol 2004; 22: 229-37. 
26 Augsten M, Hägglöf C, Peña C, Östman A. A digest on the role of the tumor microenvironment in gastrointestinal cancers. Cancer Microenviron 2010; 3: 167-76.

27 Saadi A, Shannon NB, Lao-Sirieix P et al. Stromal genes discriminate preinvasive from invasive disease, predict outcome, and highlight inflammatory pathways in digestive cancers. Proc Natl Acad Sci U S A 2010; 107: 217782.

28 Nakagawa H, Liyanarachchi S, Davuluri RV et al. Role of cancer-associated stromal fibroblasts in metastatic colon cancer to the liver and their expression profiles. Oncogene 2004; 23: 7366-77.

\section{Supporting Information}

Additional Supporting Information may be found in the online version of this article:

Fig. S1. Kaplan-Meier curves between expression levels of cancer-associated fibroblasts and M2 macrophage markers (individually) and diseasefree survival in colorectal cancer patients. 